УДК 004.6

DOI 10.18413/2687-0932-2020-47-2-402-411

\title{
МОДЕЛЬ ИНФОРМАЦИОННЫХ ПОТРЕБНОСТЕЙ МАЛОГО АГРОБИЗНЕСА В РАМКАХ ЕДИНОГО ИНФОРМАЦИОННОГО ПРОСТРАНСТВА
}

\section{MODEL OF INFORMATION NEEDS OF SMALL AGRIBUSINESS IN THE FRAMEWORK OF A SINGLE INFORMATION SPACE}

\author{
М.С. Кудашева \\ M.S. Kudasheva
}

\begin{abstract}
Пензенский государственный технологический университет, Россия, 440039, г. Пенза, проезд Байдукова/ул. Гагарина, 1a/11

Penza State Technological University, Baidukov's passage/ Gagarin street, 1A/11, Penza, 440039, Russia

E-mail: msa@penzgtu.ru
\end{abstract}

\begin{abstract}
Аннотация
Исследование информационных потребностей представителей малого агробизнеса и алгоритмов их удовлетворения является актуальной темой в условиях информатизации общества. Цель исследования - построить модель информационных потребностей малого агробизнеса, которая позволит создать концепцию единого информационного пространства для данного вида коллективных пользователей информации. В статье рассмотрены функциональные элементы модели информационных потребностей малого агробизнеса, приведена упрощенная схема движения информационных потоков. Алгоритм удовлетворения информационной потребности элементов модели представлен в виде функциональных моделей. Представленная модель является базой для развития теории информационных потребностей коллективных пользователей. Работа включает концепцию информационного комплекса малого агробизнеса, схему информационной поддержки единого информационного пространства малого агробизнеса, диаграмму Use Case. Разработка и функционирование информационного комплекса позволит обеспечить эффективность удовлетворения информационных потребностей малого агробизнеса за счет повышения доступности достоверной и актуальной информации для пользователей, а также возможности получения онлайн-консультации.
\end{abstract}

\begin{abstract}
Research of information needs of small agribusiness representatives and algorithms for their satisfaction is an urgent topic in the context of Informatization of society. The purpose of the study is to build a model of the information needs of small agribusiness, which will create a concept of a single information space for this type of collective information users. The article considers the functional elements of the model of information needs of small agribusiness, and provides a simplified diagram of information flows. The algorithm for satisfying the information needs of model elements is presented in the form of functional models. The presented model is the basis for the development of the theory of information needs of collective users. The work includes the concept of the small agribusiness information complex, the scheme of information support for the unified information space of small agribusiness, and the Use Case diagram. The development and functioning of the information complex will ensure the effectiveness of meeting the information needs of small agribusiness by increasing the availability of reliable and relevant information for users, as well as the possibility of obtaining online consultation.
\end{abstract}

Ключевые слова: информационная потребность, модель, алгоритм, элемент, функция, малый агробизнес, малая форма хозяйствования.

Keywords: information need, model, algorithm, element, function, small agribusiness, small form of management. 


\section{Введение}

Информационная потребность - это потребность субъекта в информации. Возникает она в условиях ограниченности знаний о том или ином объекте, явлении в процессе деятельности субъекта [Соколов, 2013]. И сама по себе порождает новый вид деятельности поиск путей удовлетворения информационной потребности. Субъектами информационной потребности могут выступать как отдельные потребители (например, физические лица), так и коллективные пользователи информации [Плешакова, Калюжная, 2017]. В условиях современного информационного общества вопрос удовлетворения информационных потребностей является актуальным и требует поиска новых подходов.

Процесс информатизации всех сфер экономики показал, что существуют группы пользователей информации, которые наиболее не подготовлены к вступлению в новую парадигму использования информации как ключевого ресурса бизнеса [Savinova et al., 2020]. Проведенное исследование позволило определить, что к такой группе коллективных пользователей информации можно отнести малый агробизнес, который включает крестьянские (фермерские) хозяйства, личные подсобные хозяйства, индивидуальных предпринимателей, сельскохозяйственные кооперативы. Роль малых форм хозяйствования в структуре производства сельскохозяйственной продукции занимает в среднем около 50 \% [Лямин, 2016]. При этом основными барьерами полноправного участия малого агробизнеса в информационном обществе, даже при условии его гибкости, являются: трудности в формировании адекватных информационных запросов для поиска достоверной информации, выбор информационных ресурсов, трудности в установлении информационных коммуникаций с контрагентами (поставщиками, покупателями, органами государственной власти, обслуживающей инфраструктурой), низкий уровень специальных знаний по бухгалтерскому учету и юридическому сопровождению деятельности организации [Павлов и др., 2018].

Малый агробизнес выступает как субъектом возникновения информационных потребностей, так и активным участником поиска информационных ресурсов для их удовлетворения. В связи с чем, целью настоящего исследования является построение модели информационных потребностей малого агробизнеса, которая позволит создать концепцию единого информационного пространства для данного вида коллективных пользователей информации.

\section{Объекты и методы исследования}

Объектом исследования являются информационные потребности малого агробизнеса как коллективных пользователей информации. Данный объект исследования в современной научной литературе представлен мало и поверхностно. Так в работе Лопатиной и Бекмансуровой [2014] рассматриваются больше недостатки в структуре и деятельности информационно-консультационных служб, и крайне мало говорится непосредственно о информационных потребностях представителей сельского хозяйства. Вопросы информатизации агробизнеса в научных исследованиях поднимаются чаще, однако они направлены больше на рассмотрение потребности с точки зрения обслуживающих структур агропромышленного комплекса [Кондратьева, 2017; Санду и др., 2017], создания и ведения отраслевых баз данных [Кондратьева и др., 2017], вопросов конкурентоспособности участников процесса [Федоров, 2018.]. В рамках поиска путей удовлетворения информационных потребностей малого агробизнеса нередко поднимаются темы развития технических систем информационно-консультационных служб - системы сбора информации, мониторинга производственного процесса, автоматизированной навигации [Eitzinger et al., 2019; Li et al., 2019; Plazas et al., 2019], интернета вещей [Khanna, Kaur, 2019; Popli et al., 2019] и сельскохозяйственного мобильного консалтинга [Zhimei et al., 2014; Flores et al., 2019]. В рамках настоящего исследования рассмотрим процесс удовлетворения информационных потребностей малого агробизнеса как системное явление. 
Методология исследования строится на абстрактно-логическом методе, используемом для построения теории и включающем многообразные приемы и операции, из которых будут использованы анализ, синтез, дедукция и индукция, формальная логика и гипотетическое предположение. Для построения модели информационных потребностей используем подходы информационной логистики с определением логистических потоков и взаимодействия элементов модели. Для описания алгоритма удовлетворения информационных потребностей применим функциональное моделирование, которое позволит визуализировать процесс, отобразить структуру и функции системы, а также потоки информации и материальных объектов, связывающие эти функции.

\section{Результаты и их обсуждение}

В процессе деятельности малого агробизнеса формируется стандартный набор логистических потоков: материальный, финансовый, информационный и сервисный. Ведущую роль играет материальный поток, так как он является продуктом деятельности сельскохозяйственного производства [Хмельницкая, Золотухин, 2016.]. Информационные и финансовые потоки рассматриваются как сопутствующие материальному. Исходя из цели настоящего исследования обратим пристальное внимание на формирование и прохождение информационного потока как результата удовлетворения информационной потребности. На рис. 1 представлена модель движения информационных потоков.

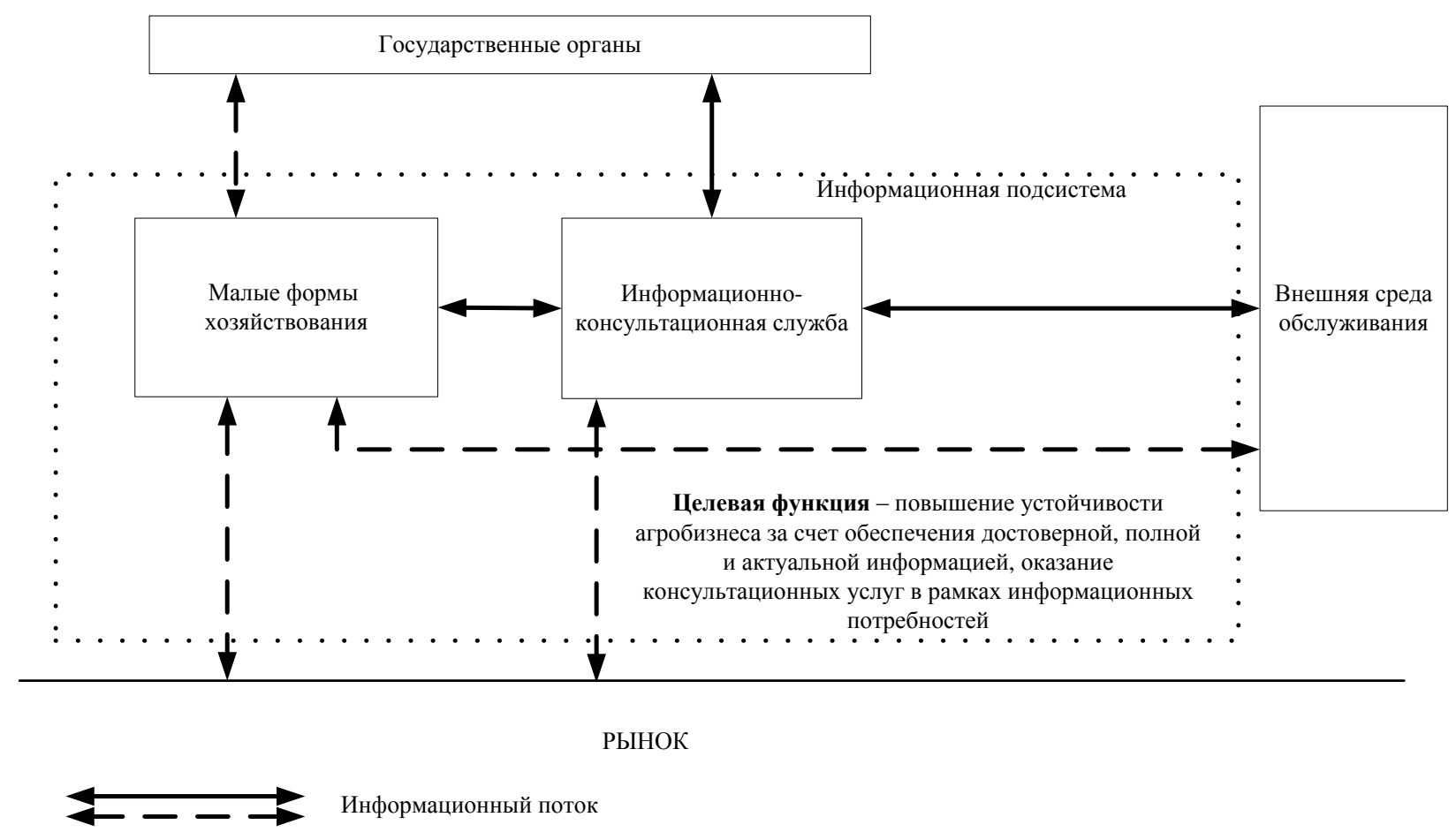

Рис. 1. Упрощенная схема движения информационных потоков в модели информационной потребности малого агробизнеса

Fig. 1. Simplified flow diagram of information flows in the model of information needs of small agribusiness

Поэлементный состав модели информационных потребностей малого агробизнеса включает:

1. Малые формы хозяйствования - центральные элементы модели, являются субъектом формирования информационной потребности и информационного потока. Основной функционал: ведение деятельности, формирование информационных запросов в соответствии с информационным интересом и потребностью, поиск источников информации и взаимодействие в рамках процесса удовлетворения информационной потребности с другими элементами системы. 
2. Информационно-консультационные службы (ИКС) - это коммерческие и некоммерческие структуры, основным назначением которых является удовлетворение информационных потребностей малых форм хозяйствования. ИКС может существовать в форме информационно-консультационных потребительских кооперативов, центров развития малого агробизнеса, консалтинговых компаний и т. п. Функции элемента - оказание услуг по бизнес-планированию, бухгалтерскому аутсорсингу, юридической и правовой поддержке, информационно-консультационному обеспечению технологических процессов.

3. Государственные органы - органы государственной власти различного уровня, агентства развития предпринимательства, основное назначение которых - организация мер финансовой, организационной и информационной поддержки развития малого агробизнеса

4. Внешняя среда обслуживания - обеспечивающие и вспомогательные организации: ревизионный союз, страховые компании, гарантийные фонды, банки, кредитные кооперативы и иные финансовые организации, научные организации, учебные заведения среднего и высшего звена, методические центры и т. п. Функции элемента: проведение ревизий, обучающих семинаров, оказание юридической помощи, предоставление необходимых финансовых ресурсов в виде кредитов (займов), предоставление поручительства и микрозаймов через гарантийный фонд, исследование информационных потребностей агробизнеса, разработка методик их эффективного удовлетворения и методических материалов, обучение. в целом.

5. Рынок - рыночная среда, конъюнктура которой влияет на деятельность всей модели

Движение информационных потоков на схеме показано в упрощенном виде для отражения общей сущности модели. Информационные потоки, отраженные пунктиром, неустойчивы, так как встречают в своем продвижении барьеры (см. рис. 1). Продвижение информационных потоков по предложенной схеме позволит за счет доступа к полной, достоверной информации и возможности реализации логистического аутсорсинга повысить удовлетворенность информационных потребностей малого агробизнеса и устойчивость финансовых и информационных потоков, связывающих малые формы хозяйствования с другими элементами модели. Кроме того, сократятся затраты на доступ к информационным ресурсам, появится возможность сконцентрировать внимание руководителя агробизнеса на основной деятельности по производству сельхозпродукции, возрастет вероятность внедрения новых технологий и техники.

Поведение элементов модели можно описать с помощью алгоритма удовлетворения информационной потребности. Этот алгоритм позволит рассмотреть узлы управления процессом и применить их для моделирования ситуации в рамках модели. На рис. 2 представлена контекстная диаграмма алгоритма удовлетворения информационной потребности.

Алгоритм поведения малой формы хозяйствования при удовлетворении информационной потребности (представлен на рис. 3) включает:

1. Осознание информационной потребности. Формируется стимул к поиску информации и информационный запрос. На данном этапе необходимо рассматривать различные ситуации возникновения информационного интереса и классификация их вариантов. Для этого оптимально использовать формулу "5W+1H" [Jia et al., 2016; Menshchikov, Lepikhin, 2018].

2 Обращение за информацией к различным источникам. Подбор источников для удовлетворения информационной потребности.

3. Действия малой формы хозяйствования в случае не обнаружения информационного источника, удовлетворяющего информационному запросу.

Эффективное информационное сопровождение модели информационных потребностей будет обеспечено реализацией единого информационного пространства малого агробизнеса как поля для информационного взаимодействия элементов модели. Технически единое информационное пространство представляет собой веб-сайт, в контент которого требуется 
включить следующие подразделы: законодательство и организационно-правовая поддержка; семинары, ярмарки, конференции; программные продукты и информационные комплексы; техника и технологии; обратная связь; форум малых форм хозяйствования. Также предлагается в качестве особого раздела включить мультимедийную базу данных, которая предназначена для хранения и доступа к информации о малых формах хозяйствования в виде текста, фото-, аудио- и видеоматериалов. Это даст возможность агробизнесу разместить рекламную информацию о своей деятельности (производимой продукции, оказываемых услугах, используемых технологиях и т. п.) с целью привлечения контрагентов к сотрудничеству.

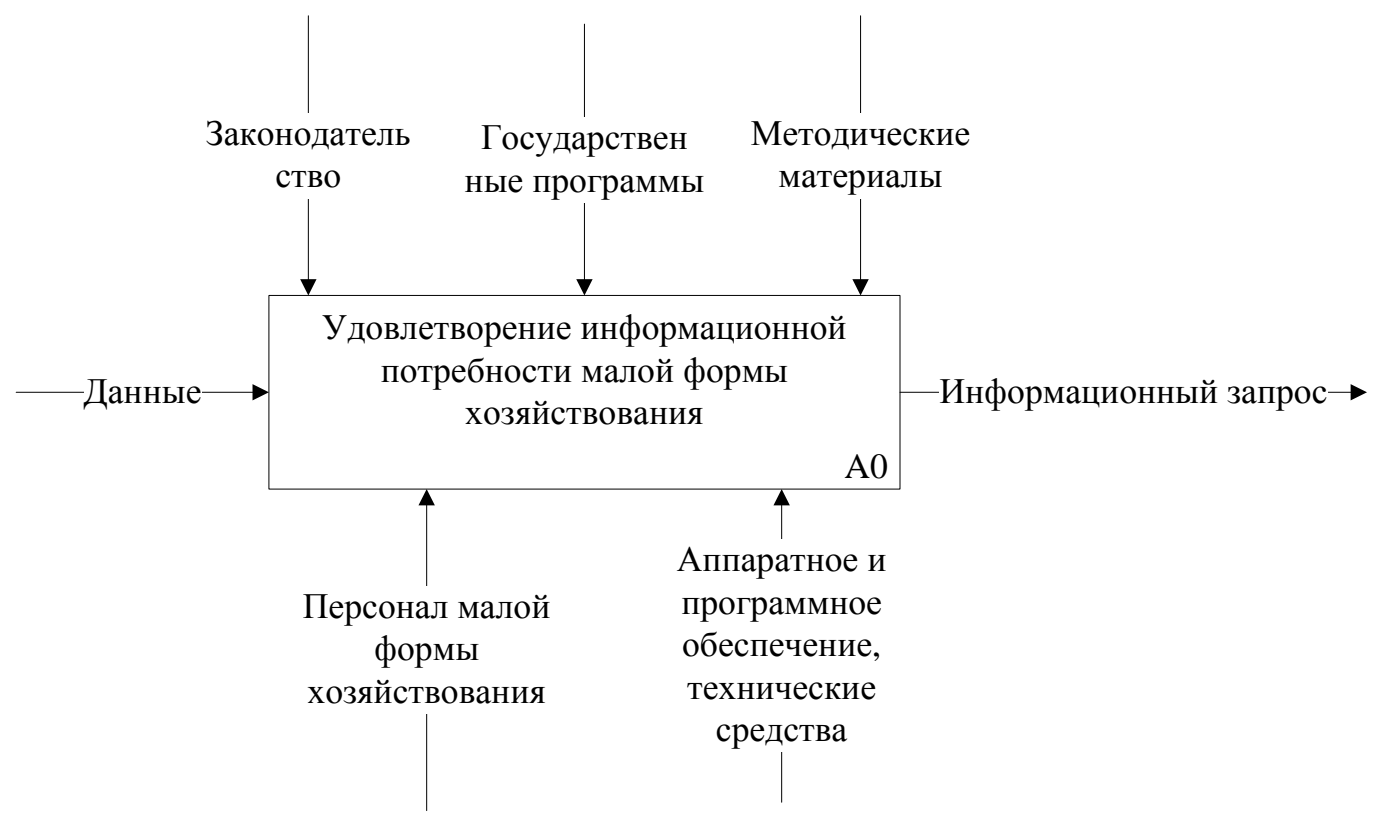

Рис. 2. Контекстная диаграмма модели

Fig. 2. Context diagram of the model

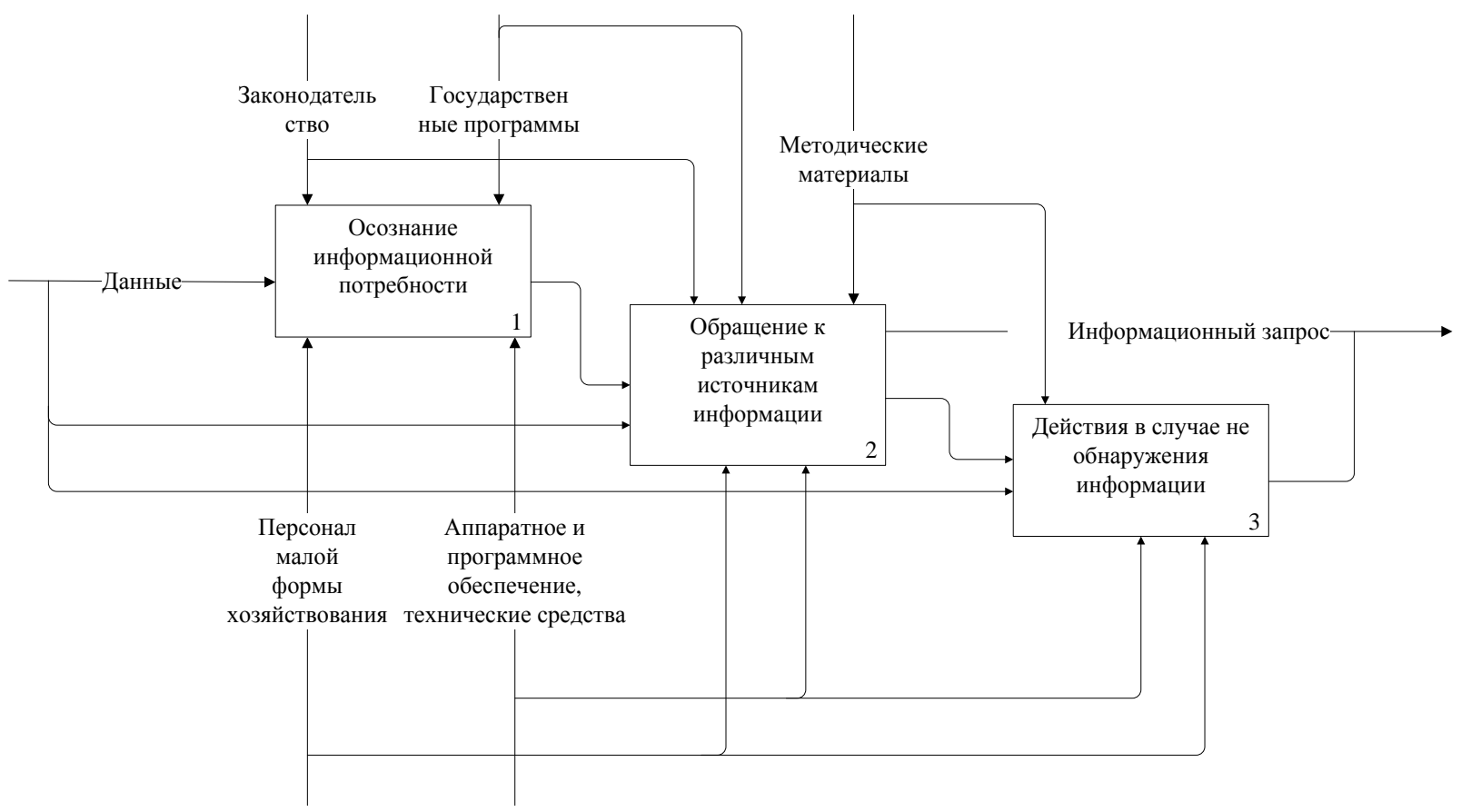

Рис. 3. Диаграмма первого уровня

Fig. 3. Diagram of the first level 
Доступ к единому информационному пространству должен осуществляться бесплатно для малых форм хозяйствования на селе, органов власти, а так же всевозможных контрагентов, которые приобретают сельхозпродукцию или поставляют технику, оборудование, сопровождающие средства и материалы для сельскохозяйственных производственных площадок. При успешной работе данного комплекса возможно применение платного входа для контрагентов после создания на базе данного веб-сайта торговой площадки с целью самоокупаемости работы единого информационного пространства малого агробизнеса.

Реализация единого информационного пространства малого агробизнеса позволит повысить эффективность удовлетворения информационной потребности агробизнеса за счет решения вопроса доступности актуальной и достоверной информации. Схема информационной поддержки единого информационного пространства малого агробизнеса представлена на рис. 4.

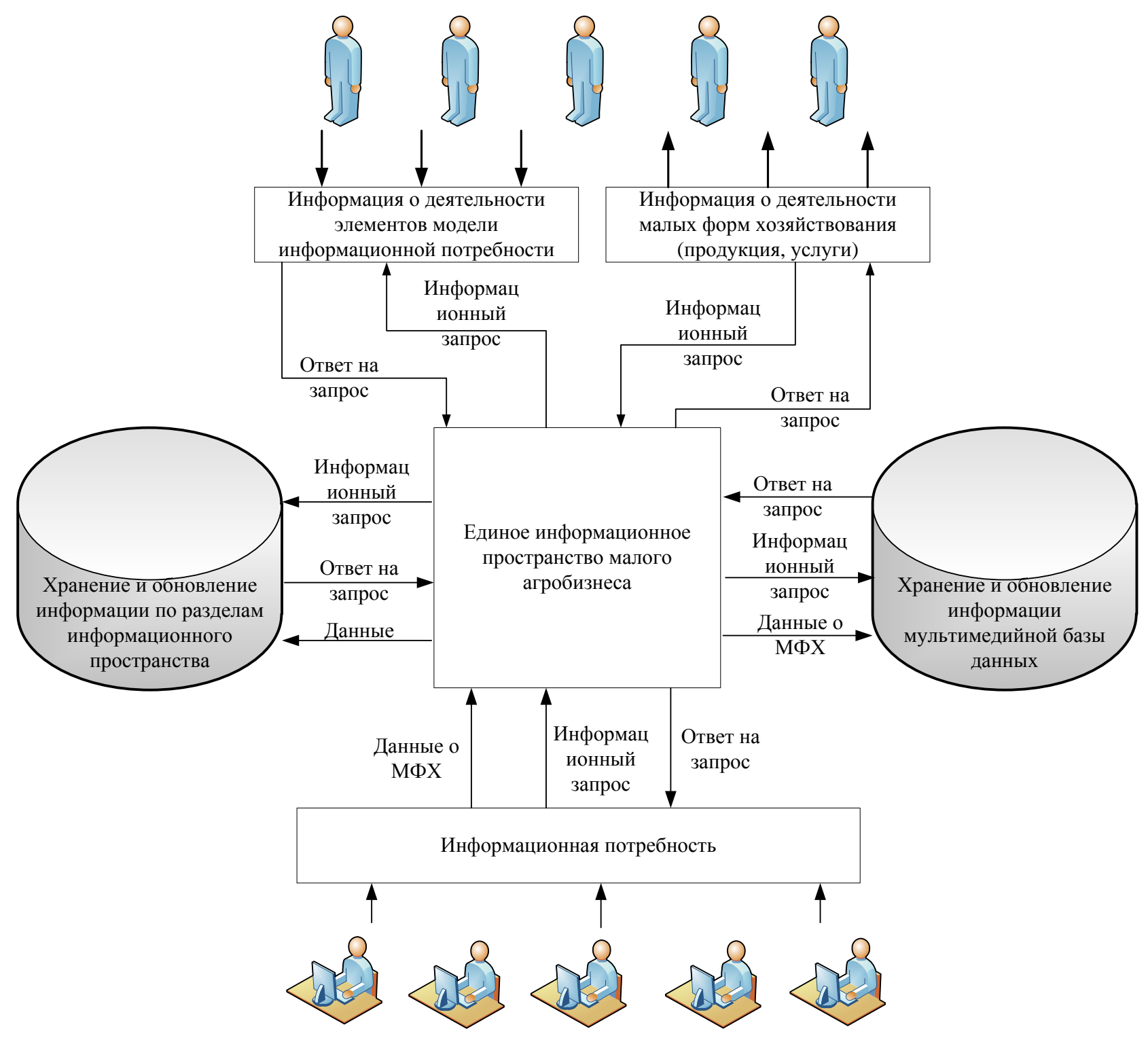

Коллективные пользователи информации - малые формы хозяйствования в агробизнесе

Рис. 4. Схема информационной поддержки единого информационного пространства малого агробизнеса

Fig. 4. Information support scheme for the unified information space for small agribusiness 
Рассмотрим процесс удовлетворения информационной потребности с помощью обращения к единому информационному пространству: представитель малой формы хозяйствования при первом обращении к веб-сайту может зарегистрироваться или просмотреть основной информационный материал без регистрации. Регистрация пользователя информационного пространства открывает для него возможность внесения данных о своей организации в мультимедийную базу данных, осуществления диалога на форуме, просмотра данных раздела «Госзакупки», входа на электронную торговую площадку. Все остальные разделы информационного пространства доступны как зарегистрированным, так и не зарегистрированным пользователям. Представитель малой формы хозяйствования может просматривать информацию по каждому из разделов сайта, воспользоваться поиском по сайту, обратиться через обратную связь с просьбой о добавлении информации или конкретным вопросом в рамках своей информационной потребности. Преимуществом использования единого информационного пространства является структурированность информации, ее актуальность и доступность, возможность консультации со специалистом ИКС.

Основываясь на предыдущем анализе сформулируем функциональные требования к веб-сайту:

1. Сайт должен позволять просматривать информацию по всем разделам сайта, осуществлять поиск по информационному запросу пользователя, регистрироваться пользователю и добавлять информацию о малой форме хозяйствования в мультимедийную базу данных, создавать тему на форуме, писать сообщения на форуме.

2. Мультимедийная база данных должна предоставлять пользователю возможность заносить не только информацию стандартной анкеты (данные об организации, ее продукции и услугах), но и добавлять текстовые, аудио- и видеофайлы. Данная информация должна проверяться администратором сайта, прежде чем стать публичной.

3. Сайт предлагает два сценария просмотра информации: для зарегистрированных пользователей и для пользователей, не прошедших регистрацию.

4. В случае отсутствия требуемой для удовлетворения информационной потребности информации или в случае возникновения трудностей с eе поиском, должна быть предусмотрена возможность задать вопрос консультанту ИКС через форму обратной связи.

5. Консультант ИКС имеет возможность зайти на данный сайт по учетной записи консультанта. На странице своей учетной записи должен видеть список заданных вопросов через форму обратной связи и иметь возможность ответить на данные вопросы с прикреплением URL-адресов, фото, текстового документа.

Учитывая описанные функциональные требования, мы можем создать диаграмму вариантов использования (рис. 5).

Анализ интернет-порталов для представителей малого агробизнеса показал, что из наиболее известных можно перечислить следующие: ФЕРМЕР.РУ (http://fermer.ru/), Информационное агентство Светич. Сайт о сельском хозяйстве. (http:// http://svetich.info/), Я-фермер.ru (http://www.ya-fermer.ru) и др. К недостаткам данных ресурсов можно отнести отсутствие полной информации по изменениям законодательной базы, мерам государственной поддержки малого агробизнеса, техническим и технологическим новинкам, не всегда есть возможность связаться со специалистом и получить консультацию. Набор представленных данных и функционал веб-сайтов не позволяет обеспечить взаимодействие элементов в рамках модели информационной потребности малого агробизнеса.

\section{Заключение}

Организация единого информационного пространства малого агробизнеса требует базы ее реализации. Предлагается для этого привлекать существующую структуру ИКС различного уровня, которая включает информационно-консультационные кооперативы, агентства по развитию предпринимательства в районах, аутсорсинговые компании.

Работу единого информационного пространства малого агробизнеса на первом этапе планируется ограничить на областном уровне. Развитие единого информационного 
пространства предполагает создание таких сайтов с информацией в каждом регионе России. Это обусловлено наличием региональной специфики ведения сельского хозяйства.

Повышение эффективности удовлетворения информационных потребностей малого агробизнеса по средствам применения единого информационного пространства обусловлено факторами повышения доступности достоверной и актуальной информации для пользователей, возможности получения онлайн-консультации с консультантом ИКС. Реализация подобного проекта тормозится отсутствием ИКС, которая возьмет на себя выполнение работ по созданию и сопровождению веб-сайта.

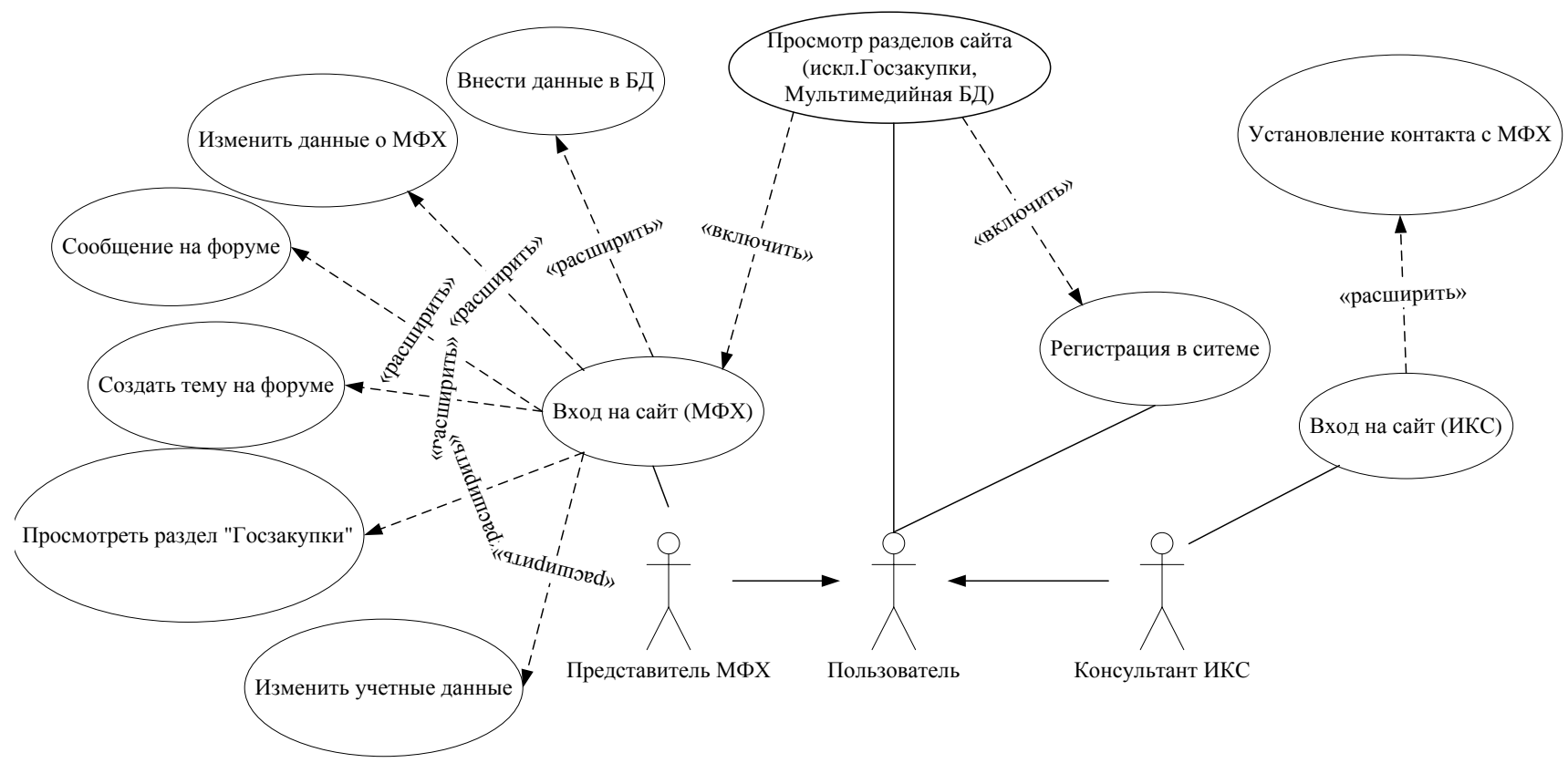

Рис. 5. Диаграмма Use Case

Fig. 5. Use Case Diagram

\section{Список литературы}

1. Кондратьева О.В. 2017. Анализ информационных потребностей и методы продвижения инновационных разработок в АПК. Техника и оборудование для села, 5: 39-41.

2. Кондратьева О.В., Федоров А.Д., Березенко Н.В., Слинько О.В. 2017. Совершенствование информационного обеспечения сельскохозяйственного производства на основе интеллектуальных информационных технологий. Синергия, 4: 68-77.

3. Лопатина С.А., Бекмансурова С.И. 2014. Выявление информационных потребностей сельскохозяйственных товаропроизводителей. Вестник Ижевской государственной сельскохозяйственной академии, 2 (39): 35-37.

4. Лямин Б.М. 2016. О роли малых форм хозяйствования в повышении устойчивости сельских территорий. Российский электронный научный журнал, 3 (21): 65-78.

5. Павлов А.Ю., Кудашева М.С., Кармышова Ю.В. 2018. Концепция интегрированной логистики как направление совершенствования информационного обеспечения деятельности сельскохозяйственных потребительских кооперативов. Российское предпринимательство, 19(2): 3863-3872.

6. Плешакова М.А., Калюжная Т.А. 2017. Обзор методов изучения информационных потребностей ученых и специалистов. Библиосфера, 3: 101-111.

7. Санду И.С., Савенко В.Г., Соколов К.О. 2017. Краудсорсинг как инструмент развития деятельности информационно-консультационных служб. Фундаментальные и прикладные исследования кооперативного сектора экономики, 6: 78-80.

8. Соколов А.В. 2013. Что есть информационная потребность? Труды Санкт-Петербургского государственного университета культуры и искусств, 197: 7-18.

9. Федоров А.Д. 2018. Цифровизация сельского хозяйства - необходимое условие повышения его конкурентоспособности. Нивы России, 5 (160): 36-39. 
10. Хмельницкая 3.Б., Золотухин С.Ю. 2016. Информационно-консультационные услуги в сфере логистики как ресурс повышения эффективности АПК. Экономика и предпринимательство, $11-$ 3 (76): 955-961.

11. Eitzinger A., Cock J., Atzmanstorfer K., Binder C.R., Läderach P., Bonilla-Findji O., Bartling M., Mwongera C., Zurita L., Jarvis A. 2019. GeoFarmer: A monitoring and feedback system for agricultural development projects. Computers and Electronics in Agriculture, 158: 109-121. DOI: 10.1016/j.compag. 2019.01.049.

12. Flores H., Villalobos J. R., Ahumada O., Uchanski M., Meneses C., Sanchez O. 2019. Use of supply chain planning tools for efficiently placing small farmers into high-value, vegetable markets. Computers and Electronics in Agriculture, 157: 205-217. DOI: 10.1007/978-3-030-40749-0_16.

13. Jia C., Yu Y.T., Cai Y., Tse T.H. 2016. 5W+1H pattern: a perspective of systematic mapping studies and a case study on cloud software testing. Journal of Systems and Software, 116: 206-219. DOI: 10.1016/j.jss.2015.01.058.

14. Khanna A., Kaur S. 2019. Evolution of internet of things (IoT) and its significant impact in the field of precision agriculture. Computers and Electronics in Agriculture, 157: 218-231. DOI:10.1016/ j.compag.2018.12.039.

15. Li S., Xu H., Ji Y., Cao R., Zhang M., Li H. 2019. Development of a following agricultural machinery automatic navigation system. Computers and Electronics in Agriculture, 158: 335-344.

16. Menshchikov M., Lepikhin T. 2018. 5W+1H static analysis report quality measure. Communications in Computer and Information Science, 779: 114-126. DOI: 10.1007/978-3-319-71734-0_10.

17. Plazas J.E., Bimonte S., De Sousa G., Corrales J.C. 2019. Data-centric UML profile for wireless sensors: Application to smart farming. International Journal of Agricultural and Environmental Information Systems, 10 (2): 21-48. DOI:10.4018/ijaeis.2019040102.

18. Popli S., Jha R. K., Jain S. 2019. A survey on energy efficient narrowband internet of things (NBIoT): Architecture, application and challenges. IEEE Access, 7: 16739-16776.

19. Savinova V.A., Zhegalova E.V., Semernina J.V., Kozlova A.S. 2020. Problems of the development of the digital economy at the regional level. Advances in Intelligent Systems and Computing, 908: 260-268. DOI:10.1007/978-3-030-11367-4_25.

20. Zhimei L., Changshou L., Junfeng Z. 2014. Research on the development and preliminary application of 12396 new rural sci-tech service hotline wechat public platform. Open Automation and Control Systems Journal, 6 (1): 839-843. DOI: 10.2174/1874444301406010839.

\section{References}

1. Kondratieva O.V. 2017. Analiz informatsionnykh potrebnostei i metody prodvizheniya innovatsionnykh razrabotok v APK [Analysis of Information Needs and Methods of Promotion of Innovation Developments in Agribusiness]. Tekhnika i oborudovanie dlya sela, 5: 39-41.

2. Kondratyeva O.V., Fedorov A.D., Berezenko N.V., Slin'ko O.V. 2017. Enhancement of information support of agricultural production on the basis of intellectual information technologies. Sinergiya, 4: 68-77. (in Russian).

3. Lopatina S.A., Bekmansurova S.I. 2014. Vyyavlenie informatsionnykh potrebnostei sel'skokhozyaistvennykh tovaroproizvoditelei [Identification of information needs of agricultural producers].The Bulletin of Izhevsk State Agricultural Academy, 2 (39): 35-37.

4. Lyamin B.M. 2016. Role of small farms in enhancing sustainable development of rural areas. Russian electronic scientific magazine, 3 (21): 65-78 (in Russian).

5. Pavlov A.Yu., Kudasheva M.S., Karmyshova Yu.V. The concept of integrated logistics as a direction of improvement of information support of agricultural consumer cooperatives. Russian journal of entrepreneurship, 19(2): 3863-3872 (in Russian).

6. Pleshakova M.A., Kalyuzhnaya T.A. 2017. Methods review to study information needs of scientists and specialists. BIBLIOSPHERE, 3: 101-111 (in Russian).

7. Sandu I.S., Savenko V.G., Sokolov K.O. 2017. Crowdsourcing as instrument of development of activity of information and consulting services. Fundamental and applied researches of the cooperative sector of the economy, 6: 78-80 (in Russian).

8. Sokolov A.V. 2013. Chto est' informatsionnaya potrebnost'? [What is the information need?]. Trudy Sankt-Peterburgskogo gosudarstvennogo universiteta kul'tury i iskusstv, 197: 7-18. 
9. Fedorov A.D. 2018. Tsifrovizatsiya sel'skogo khozyaistva - neobkhodimoe uslovie povysheniya ego konkurentosposobnosti [Digitalization of agriculture is a necessary condition for increasing its competitiveness]. Nivy Rossii, 5 (160): 36-39.

10. Khmel'nitskaya Z.B., Zolotukhin S.Yu. 2016. Information and consulting services in the field of logistics as a resource for increasing efficiency of agriculture. Journal of Economy and entrepreneurship, 113 (76): 955-961 (in Russian).

11. Eitzinger A., Cock J., Atzmanstorfer K., Binder C.R., Läderach P., Bonilla-Findji O., Bartling M., Mwongera C., Zurita L., Jarvis A. 2019. GeoFarmer: A monitoring and feedback system for agricultural development projects. Computers and Electronics in Agriculture, 158: 109-121. DOI: 10.1016/ j.compag.2019.01.049.

12. Flores H., Villalobos J. R., Ahumada O., Uchanski M., Meneses C., Sanchez O. 2019. Use of supply chain planning tools for efficiently placing small farmers into high-value, vegetable markets. Computers and Electronics in Agriculture, 157: 205-217. DOI: 10.1007/978-3-030-40749-0_16.

13. Jia C., Yu Y.T., Cai Y., Tse T.H. 2016. 5W+1H pattern: a perspective of systematic mapping studies and a case study on cloud software testing. Journal of Systems and Software, 116: 206-219. DOI: 10.1016/j.jss.2015.01.058.

14. Khanna A., Kaur S. 2019. Evolution of internet of things (IoT) and its significant impact in the field of precision agriculture. Computers and Electronics in Agriculture, 157: 218-231. DOI:10.1016/ j.compag.2018.12.039.

15. Li S., Xu H., Ji Y., Cao R., Zhang M., Li H. 2019. Development of a following agricultural machinery automatic navigation system. Computers and Electronics in Agriculture, 158: 335-344.

16. Menshchikov M., Lepikhin T. 2018. 5W+1H static analysis report quality measure. Communications in Computer and Information Science, 779: 114-126. DOI: 10.1007/978-3-319-71734-0_10.

17. Plazas J.E., Bimonte S., De Sousa G., Corrales J.C. 2019. Data-centric UML profile for wireless sensors: Application to smart farming. International Journal of Agricultural and Environmental Information Systems, 10 (2): 21-48. DOI:10.4018/ijaeis.2019040102.

18. Popli S., Jha R. K., Jain S. 2019. A survey on energy efficient narrowband internet of things (NBIoT): Architecture, application and challenges. IEEE Access, 7: 16739-16776.

19. Savinova V.A., Zhegalova E.V., Semernina J.V., Kozlova A.S. 2020. Problems of the development of the digital economy at the regional level. Advances in Intelligent Systems and Computing, 908: 260-268. DOI:10.1007/978-3-030-11367-4_25.

20. Zhimei L., Changshou L., Junfeng Z. 2014. Research on the development and preliminary application of 12396 new rural sci-tech service hotline wechat public platform. Open Automation and Control Systems Journal, 6 (1): 839-843. DOI: 10.2174/18744444301406010839.

\section{Ссылка для цитирования статьи For citation}

Кудашева М.С. 2020. Модель информационных потребностей малого агробизнеса в рамках единого информационного пространства. Экономика. Информатика. 47 (2): 402-411. DOI: 10.18413/2687-0932-2020-47-2-402-411.

Kudasheva M.S. 2020. Model of information needs of small agribusiness in the framework of a single information space. Economics. Information technologies. 47 (2): $402-411$ (in Russian). DOI: 10.18413/26870932-2020-47-2-402-411. 